\title{
Molecular characterisation of group A streptococci from invasive and non-invasive disease episodes in Belgium during 1993-1994
}

\author{
P. DESCHEEMAEKER, F. VAN LOOCK*, M. HAUCHECORNE, P. VANDAMME and H. GOOSSENS
}

National Reference Centre for GAS, Department of Microbiology, University Hospital Antwerp, Universitaire Instelling Antwerpen, Antwerp and * Scientific Institute for Public Health - Louis Pasteur, Brussels, Belgium

\begin{abstract}
Five hundred clinical group A streptococcal (GAS) isolates were collected in Belgium during the period 1 Nov. 1993 to 31 Oct. 1994. Clinical and laboratory data were recorded and isolates were characterised. The presence of the genes encoding streptococcal pyrogenic exotoxin types A (speA), B (speB), C (speC), F (speF) and streptococcal superantigen (ssa) were determined by PCR to target specific sequences. These isolates were also emm-typed and analysed by pulsed-field gel electrophoresis (PFGE) of genomic macrorestriction fragments with the enzyme SmaI. In total, 136 unrelated GAS PFGE types were identified and genetic diversity was clearly demonstrated. Two GAS PFGE types predominated; a first PFGE type comprised 66 (13.2\%) emm 1 isolates characterised by $\mathrm{speA}^{+}, \mathrm{speB}^{+}, \mathrm{speC}^{-}, \mathrm{speF}^{+}$and $\mathrm{ssa}^{-}$; the second PFGE type comprised $44(8.8 \%)$ emm 12 isolates characterised by $\mathrm{speA}^{-}, \mathrm{speB}^{+}, \mathrm{speC}^{+}$(or $\mathrm{speC}^{-}$), speF $\mathrm{F}^{+}$and $\mathrm{ssa}^{-}$. Indistinguishable PFGE types were observed among both invasive and non-invasive isolates. Ten different PFGE types were found among 11 streptococcal toxic shock syndrome (STSS) isolates, and five of these lacked speA. Twenty-five (34.7\%) of 72 invasive isolates gave negative results for speA, speC and ssa. This retrospective study confirmed the observation that the dissemination of one specific clone cannot be associated with invasive GAS disease and posed a question regarding the role of SPE A as a major virulence factor. Other streptococcal virulence factors in conjunction with host factors may determine the outcome of invasive GAS infection.
\end{abstract}

\section{Introduction}

Streptococcus pyogenes is one of the most common and ubiquitous human pathogens and is responsible for a wide range of infections varying from clinically mild infections (pharyngitis, impetigo, scarlet fever, etc.), to severe life-threatening infections (sepsis, necrotising fasciitis, toxic shock syndrome), to non-suppurative sequelae such as rheumatic fever and acute glomerulonephritis [1-5].

In the late 1980s, reports on the resurgence of severe group A streptococcal (GAS) infection [3, 6-9] resulted in an increased awareness and interest in this

Received 24 March 1999; revised version accepted 20 Sept. 1999.

Corresponding author: Dr P. Descheemaeker (e-mail: Patrick. Descheemaeker@azbrugge.be). organism. The introduction of a world-wide dispersed GAS clone characterised by $M$ serotype 1 and genotype ET1/RFLP type 1a (as defined by Musser et al. [10]) into an immunological naive population was thought to play a role in the contemporary resurgence of severe invasive disease [10]. Recently, highly discriminative molecular techniques, such as FAFLP [11] and sic gene sequencing [12], showed some microheterogeneity among these M1 isolates. However, when isolates from invasive and non-invasive infections were studied, there were no streptococcal virulence factors or specific clones that appeared to be directly associated with invasive disease $[13,14]$.

The objectives of the present study were to evaluate the genetic diversity and the prevalence of the ET1/RFLP 1a subclone among a collection of 500 invasive and non-invasive Belgian GAS isolates and to evaluate the relationship between severity of disease, presence of superantigen genes and different genotypic clones. 


\section{Materials and methods}

\section{Bacterial strains}

During the period 1 Nov. 1993 to 31 Oct. 1994, 1720 GAS isolates were collected from 89 diagnostic laboratories geographically distributed within Belgium together with a completed questionnaire which included demographic, clinical, epidemiological and laboratory information. The isolates were categorised according to their site of isolation, the clinical syndromes and complications associated with the infection. A total of 500 isolates was selected for this present study. Category 1 comprised a random sample of 102 of the 1322 pharyngeal GAS isolates. Category 2 included all 326 GAS isolates from non-sterile sites collected from patients with non-invasive infections other than pharyngitis, which included impetigo, otitis media, vaginitis and sinusitis. Category 3 included 72 GAS isolates from sterile sites (blood, sterile body fluids, abscesses or tissue) of patients with invasive infections. Based upon the case definition of streptococcal toxic shock syndrome (STSS) [15], 17 cases of STSS were reported, 11 of which could be included as definite cases (four isolates were not submitted to this reference laboratory). Fifty-one reference strains were included for comparison.

\section{Genomic diversity}

A total of 500 clinical isolates and 51 reference strains was examined by pulsed-field gel electrophoresis (PFGE) with the restriction enzyme SmaI as described elsewhere [16]. The emm genotype was determined by hybridisation of the denatured emm gene amplicon with a panel of $26 \mathrm{emm}$-type specific probes in a reversed line blotting system according to the genotyping method described by Kaufhold et al. [17].

\section{Distribution of virulence genes}

The superantigen genes, spe $A$, speB and speC, were detected as described by Black et al. [31], speF as described by Norby-Teglund et al. [32] and ssa as described by Reda et al. [14].

\section{Results and discussion}

\section{Genomic diversity}

Visual and computerised analysis of the Sma I patterns revealed 136 unrelated GAS PFGE types, when three or more band differences between two patterns was used as a criterion to define a PFGE type [18], which demonstrated a very high genetic variability amongst GAS in Belgium. The 10 most frequently found PFGE types comprised $46 \%$ of the isolates studied (Table 1 ). Twelve of the 551 DNA samples were either not digested or only partly digested by SmaI and were characterised by a band of undigested genomic DNA (Fig. 1, lane 3). It was interesting to note that these 12 strains were erythromycin-resistant, expressing the $\mathrm{M}$ resistance phenotype $[19,20]$.

The largest cluster of strains, designated PFGE type 01, and characterised by emm-type 1, constituted $13.2 \%$ $(\mathrm{n}=66)$ of the clinical isolates examined (Table 1) and were characterised by a unique Sma I pattern (Fig. 1, lanes 1 and 2). This PFGE type was found to be identical to the world-wide disseminated M-type 1, ET1/RFLP1a clone defined by Musser et al. [10]. A second cluster of strains, designated PFGE type 02 and characterised by emm-type 12, constituted $8.8 \%$ $(\mathrm{n}=44)$ of the clinical isolates (Table 1), and was characterised by two highly related SmaI patterns differing in two band positions (Fig. 1, lanes 3-7). These two PFGE types were also recently shown to be the predominant genotypes found in France [21]. In Canada, during the period 1992-1993, M-type 1 and

Table 1. Distribution of the most prevalent Sma I PFGE types among the different categories

\begin{tabular}{|c|c|c|c|c|c|c|c|c|c|}
\hline \multirow[b]{2}{*}{ Genotype* } & \multicolumn{4}{|c|}{ Percent (number) of strains } & \multirow[b]{2}{*}{ emm type } & \multicolumn{3}{|c|}{ Superantigen genes* } & \multirow[b]{2}{*}{ OR $(95 \% \mathrm{CL})^{\S}$} \\
\hline & Category $^{\dagger} 1$ & Category $^{\dagger} 2$ & Category $^{\dagger} 3$ & Total & & speA & speC & $s s a$ & \\
\hline 01 & $13.7(14)$ & $13.8(45)$ & 9.7 (7) & $13.2(66)$ & emm 1 & + & - & - & $0.67(0.25-1.57)$ \\
\hline 02 & $18.7(17)$ & $5.2(17)$ & $13.8(10)$ & $8.8(44)$ & emm 12 & - & $+/-$ & - & $1.87(0.78-4.11)$ \\
\hline 03 & $4.9(5)$ & $5.5(18)$ & $1.4(1)$ & $4.8(24)$ & emm 4 & - & + & + & $0.25(0.01-1.58)$ \\
\hline 04 & $6.9(7)$ & $3.4(11)$ & $8.3(6)$ & $4.8(24)$ & NT & $+/-$ & $+/-$ & - & $2.07(0.65-5.69)$ \\
\hline 05 & $2.0(2)$ & $2.8(9)$ & $1.4(1)$ & $2.4(12)$ & emm 3 & + & $+/-$ & + & $0.53(0.01-3.78)$ \\
\hline 06 & $0.0(0)$ & $3.7(12)$ & $1.4(1)$ & $2.6(13)$ & emm 6 & $+/-$ & $+1-$ & - & $0.49(0.01-3.40)$ \\
\hline 07 & $1.0(1)$ & $2.8(9)$ & $5.6(4)$ & $2.8(14)$ & emm 11 & - & + & - & $2.46(0.55-8.81)$ \\
\hline 08 & 3.9 (4) & $2.8(9)$ & $2.8(2)$ & $3.0(15)$ & NT & - & + & - & $0.91(0.10-4.16)$ \\
\hline 09 & $1.0(1)$ & $2.2(7)$ & $2.8(2)$ & $2.0(10)$ & NT & - & - & - & $1.50(0.15-7.76)$ \\
\hline 10 & $2.0(2)$ & $1.5(5)$ & $1.4(1)$ & $1.6(8)$ & emm 9 & - & - & + & $0.85(0.02-6.76)$ \\
\hline Other & $48.0(49)$ & $56.4(184)$ & $51.4(37)$ & $54.0(270)$ & $\ldots$ & $\cdots$ & $\cdots$ & $\cdots$ & $0.88(0.52-1.51)$ \\
\hline Total & 102 & 326 & 72 & 500 & & & & & \\
\hline
\end{tabular}

NT, non-typable with panel of probes examined.

*PFGE types delineated by SmaI mr analysis.

${ }^{\dagger}$ Categories as defined in the text.

\$PCR detection of superantigen genes: +, present; -, absent.

$\S$ Odds ratios and exact confidence limits were computed in a univariate analysis comparing categories 1 and 2 (reference category) against category 3 . 


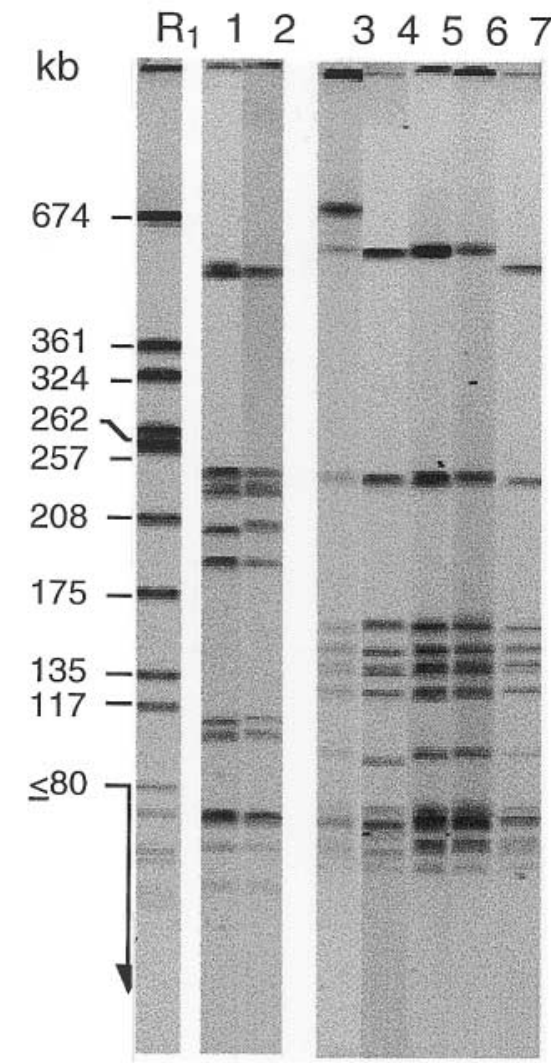

Fig. 1. PFGE patterns of SmaI-digested chromosomal DNA from genotype 01 (lanes 1 and 2) and genotype 02 (3-7) strains. The PFGE profiles were obtained after analysis with the computer software GelCompar version 4.0. (Applied Maths, Kortrijk, Belgium). $\mathbf{R}_{\mathbf{1}}$, Staphylococcus aureus NCTC 8325 reference type strain for Sma I digests.

M-type 12 strains also predominated [22], although PFGE data were not presented in the study. Each of the other PFGE types comprised $<5 \%$ of the clinical isolates examined (Table 1). The high frequency by which these two clones were found may be explained by poor herd immunity towards these PFGE types [10], or alternatively these strains may possess characteristics that contribute to their survival in man. The 11 STSS strains belonged to 10 different SmaI PFGE types, which included PFGE types 01 (two strains), 04 and 09.

Among the 10 most common PFGE types, a single genotype was not associated with severe invasive disease (category 3, Table 1). PFGE type 01, which had been suggested to be responsible in part for the increasing number of severe invasive infections seen since the late 1980s [23,24], was not significantly associated with severe invasive disease. Furthermore, indistinguishable GAS isolates were found among the three categories of strains. Similarly, Muotiala et al. [25] and Kiska et al. [26] observed a concomitant increase of serotype T1M1 isolates among individuals with bacteraemic and pharyngeal infections and Kazumitsu et al. [27] also found identical fingerprints among both invasive and non-invasive isolates.

Thus, the distribution of indistinguishable PFGE types and the clonal variability among invasive and noninvasive isolates does not support the existence of a more invasive $S$. pyogenes clone. In many studies on STSS, or other invasive GAS infections, throat isolates from pharyngitis cases were not or very rarely included and epidemiological typing was not undertaken [23, 28-30]. Therefore, conclusions on clonal relationship and association of particular clones with invasive disease may be biased. The present study and others [25] showed that studying these so-called non-invasive isolates in comparison with invasive isolates may be valuable for epidemiological purposes and may result in a better understanding of the pathogenetic properties of invasive GAS strains.

\section{Distribution of virulence genes}

The chromosomally encoded genes speB and speF were detected in $100 \%$ of the isolates. The speC gene was most frequently detected in every category described (Table 2). A study of the presence or the

Table 2. Distribution of the streptococcal virulence genes among 500 Belgian GAS isolates

\begin{tabular}{|c|c|c|c|c|c|c|c|}
\hline \multirow{2}{*}{\multicolumn{3}{|c|}{ Superantigen gene detection* }} & \multicolumn{3}{|c|}{ Percent (number) of strains in category } & \multirow{2}{*}{$\begin{array}{l}\text { Mean percent } \\
\text { (total number) }\end{array}$} & \multirow[b]{2}{*}{ OR $(95 \% \mathrm{CL})^{\dagger}$} \\
\hline & & & 1 & 2 & 3 & & \\
\hline \multirow[b]{4}{*}{ 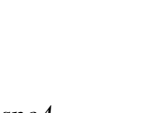 } & speA $^{+}$ & & $20.6 \%(21)$ & $25.2 \%(82)$ & $29.2 \%(21)$ & $24.8 \%(124)$ & $130(0.71-2.32)$ \\
\hline & speC $^{+}$ & & $53.9 \%(55)$ & $55.5 \%(181)$ & $44.4 \%(32)$ & $53.6 \%(268)$ & $0.65(0.38-1.11)$ \\
\hline & $s s a^{+}$ & & $25.5 \%(26)$ & $34.4 \%(112)$ & $15.3 \%(11)$ & $29.8 \%(149)$ & $0.38(0.17-0.76)$ \\
\hline & speC & $s s a^{\ddagger}$ & & & & & \\
\hline- & - & - & $18.6 \%(19)$ & $16.6 \%(54)$ & $34.7 \%(24)$ & $19.6 \%(98)$ & $2.59(1.43-4.60)$ \\
\hline- & - & + & $7.8 \%(8)$ & $8.6 \%(28)$ & $5.6 \%(4)$ & $8.0 \%(40)$ & $0.64(0.16-1.87)$ \\
\hline- & + & - & $38.2 \%$ (39) & $30.1 \%(98)$ & $23.6 \%(17)$ & $30.8 \%(154)$ & $0.66(0.34-1.20)$ \\
\hline- & + & + & $14.7 \%(15)$ & $19.6 \%(64)$ & $6.9 \%(5)$ & $16.8 \%(84)$ & $0.33(0.10-0.85)$ \\
\hline+ & - & - & $16.7 \%(17)$ & $14.7 \%(48)$ & $12.5 \%(9)$ & $14.8 \%(74)$ & $0.80(0.33-1.72)$ \\
\hline+ & - & + & $2.9 \%(3)$ & $4.6 \%(15)$ & $1.4 \%(1)$ & $3.8 \%$ (19) & $0.32(0.01-2.10)$ \\
\hline+ & + & - & $1.0 \%(1)$ & $4.3 \%(14)$ & $13.9 \%(10)$ & $5.0 \%(25)$ & $7.65(2.83-19.61)$ \\
\hline+ & + & + & $0 \%(0)$ & $1.5 \%(5)$ & $1.4 \%(1)$ & $1.2 \%(6)$ & $1.19(0.02-10.87)$ \\
\hline Total & & & 102 & 326 & 72 & 500 & \\
\hline
\end{tabular}

* Percentage of strains comprising the genes speA, speC or ssa.

$\dagger$ Odds ratios and exact confidence limits were computed in a univariate analysis comparing categories 1 and 2 (reference category) against category 3 .

Tercentage of strains comprising the different genes: +, present; -, absent. 
absence of the three genes speA, speC and ssa combined (Table 2) showed a statistically significant association between strains that lacked these three genes and severe invasive infection (category 3). Also, the presence of the genes speA and speC and the absence of the gene ssa appeared to correlate with severe invasive infection, although the number of strains examined was limited. A negative association was observed between the absence of speA and the presence of speC and ssa and severe invasive infections, although this was not confirmed when category 1 strains were compared to categories 2 and 3. Strains harbouring all three genes were rarely detected.

PFGE type 01 was characterised by the exotoxin gene profile $s p e A^{+}, s p e B^{+}, s p e C^{-}, s p e F^{+}$and $s s a^{-}$(Table $1)$. Among genotype 02 strains, the following exotoxin gene profile was found; $s p e A^{-}, s p e B^{+}, s p e C^{+}$or spe $^{-}, \mathrm{speF}^{+}$and $\mathrm{ssa}^{-}$(Table 1). Genotype 02 strains lacked the bacteriophage-encoded gene speC and typically demonstrated in their SmaI profile a band of a lower fragment size than the genotype 02 strains that harboured the speC gene (Fig. 1, lane 7).

Four different exotoxin gene profiles were observed among the 11 STSS isolates, with the majority (five strains) lacking the three genes speA, speC and ssa. None of the STSS strains possessed the ssa gene.

The streptococcal pyrogenic exotoxins or combinations of toxins may be responsible for the invasiveness of certain GAS clones. The results of the present study did not confirm the reported association between the exotoxin SPE A and severe invasive disease [9, $23,33,34]$. Indeed, $70.8 \%$ of category 3 strains did not harbour the gene speA, and the genes speC and ssa were less frequent in category 3 than in the other categories (Table 2). Moreover, in $34.7 \%$ of the isolates collected from patients with severe GAS infections (category 3) the study failed to detect the genes speA, speC and ssa (Table 2). This corroborates the results of the study by Hsueh et al. [35], who reported that the presence of speA, speC or speF did not implicate any particular clinical syndrome in patients with invasive GAS disease in Taiwan. These data suggest that other factors or superantigens may be responsible for invasive infection. This was also suggested by Watanabe et al. [36], who demonstrated that specific $\mathrm{V} \beta$ depletion in the sera of patients who had STSS and other severe invasive infections did not correlate with the in-vitro pattern of any previously characterised streptococcal superantigen [37]. Recently a novel exotoxin - streptococcal mitogenic exotoxin Z (SME Z) - was identified [38], stressing the existence of yet another unknown, possibly virulence-related exotoxin. Clones which lacked the three genes speA, speC and ssa were also found among both invasive and noninvasive isolates (data not shown). This further highlights the fact that the outcome of a GAS infection is not purely strain related, but is a combination of several factors amongst which host immunity and factors regulating the expression of the exotoxin genes may be of major importance [39].

In summary, the data from the present study demonstrated a very high genetic diversity among $S$. pyogenes isolates in Belgium. A limited number of clones occurred with a high frequency, although they were equally distributed among the invasive and noninvasive isolates. These findings do not support the existence of 'a more invasive' clone rather than multiple opportunist virulent clones which perhaps become more invasive in conjunction with the immune status of the host.

Furthermore, other as yet unknown bacterial characteristics may also play a role in severe streptococcal infections, as GAS without the superantigen genes speA, speC and ssa were able to cause invasive disease. This highlights the potential role of SPE B. These findings also suggest the need for further characterisation of both invasive and non-invasive isolates, and for studying the regulation of exotoxin expression and the interaction with host factors. Furthermore, there is a need for constant microbiological and epidemiological surveillance and pathogenic studies.

P.V. is indebted to the Fund for Scientific Research - Flanders (Belgium) for a position as a postdoctoral research fellow and M.H. for a position as technical assistant. We thank all Belgian clinical laboratories for the submission of the strains examined.

\section{References}

1. Bisno AL. Group A streptococcal infections and acute rheumatic fever. $N$ Engl J Med 1991; 325: 783-793.

2. Bisno AL, Stevens DL. Streptococcal infections of skin and soft tissues. $N$ Engl J Med 1996; 334: 240-245.

3. Demers B, Simor AE, Vellend $\mathrm{H}$ et al. Severe invasive group A streptococcal infections in Ontario, Canada: 1987-1991. Clin Infect Dis 1993; 16: 792-800.

4. Denny FW. A 45-year perspective on the streptococcus and rheumatic fever: The Edward $H$. Kass lecture in infectious disease history. Clin Infect Dis 1994; 19: 1110-1122.

5. Stevens DL. Invasive group A streptococcus infections. Clin Infect Dis 1992; 14: 2-13.

6. Holm SE, Norrby A, Bergholm A-M, Norgren M. Aspects of pathogenesis of serious group A streptococcal infections in Sweden, 1988-1989. J Infect Dis 1992; 166: 31-37.

7. Hoge CW, Schwartz B, Talkington DF, Breiman RF, MacNeill EM, Englender SJ. The changing epidemiology of invasive group A streptococcal infections and the emergence of streptococcal toxic shock-like syndrome. JAMA 1993; 269: 384-389.

8. Katz AR, Morens DM. Severe streptococcal infections in historical perspective. Clin Infect Dis 1992; 14: 298-307.

9. Stevens DL, Tanner MH, Winship J et al. Severe group A streptococcal infections associated with a toxic shock-like syndrome and scarlet fever toxin A. N Engl J Med 1989; 321: $1-7$.

10. Musser JM, Kapur V, Szeto J, Pan X, Swanson DS, Martin DR. Genetic diversity and relationships among Streptococcus pyogenes strains expressing serotype M1 protein: recent international spread of a subclone causing episodes of invasive disease. Infect Immun 1995; 63: 994-1003.

11. Desai M, Efstratiou A, George R, Stanley J. High-resolution genotyping of Streptococcus pyogenes serotype M1 isolates by 
fluorescent amplified-fragment length polymorphism analysis. J Clin Microbiol 1999; 37: 1948-1952.

12. Hoe N, Nakashima K, Grigsby D et al. Rapid molecular genetic subtyping of serotype M1 group A Streptococcus strains. Emerg Infect Dis 1999; 5: 254-263.

13. Norrby-Teglund A, McGeer A, Low D, Kotb M. Superantigenicity and $\mathrm{V} \beta$-specificity of culture supernatants from streptococcal toxic shock syndrome Streptococcus pyogenes isolates. In: XIIIth Lancefield International Symposium on Streptococci and Streptococcal Diseases, 16-20 Sept. 1996. Montreuil-Sous-Bois, France, Orane Communication Graphique. 1996.

14. Reda KB, Kapur V, Goela D, Lamphear JG, Musser JM, Rich RR. Phylogenetic distribution of streptococcal superantigen SSA allelic variants provides evidence for horizontal transfer of ssa within Streptococcus pyogenes. Infect Immun 1996; 64: $1161-1165$.

15. The Working Group on Severe Streptococcal Infections. Defining the group A streptococcal toxic shock syndrome. Rationale and consensus definition. JAMA 1993; 269: 390-391.

16. Descheemaeker P, Lammens C, Pot B, Vandamme P, Goossens $\mathrm{H}$. Evaluation of arbitrarily primed PCR analysis and pulsedfield gel electrophoresis of large genomic DNA fragments for identification of enterococci important in human medicine. Int $J$ Syst Bacteriol 1997; 47: 555-561.

17. Kaufhold A, Podbielski A, Baumgarten G, Blokpoel M, Top J, Schouls L. Rapid typing of group A streptococci by the use of DNA amplification and non-radioactive allele-specific oligonucleotide probes. FEMS Microbiol Lett 1994; 119: 19-26.

18. Tenover FC, Arbeit RD, Goering RV et al. Interpreting chromosomal DNA restriction patterns produced by pulsedfield gel electrophoresis: criteria for bacterial strain typing. J Clin Microbiol 1995; 33: 2233-2239.

19. Goossens H, Chapelle S, Hauchecorne M, Wijdooghe M, Descheemaeker P. Characterization of macrolide resistance among group A Streptococcus in Belgium [abstract C-14]. In: Program and Abstracts of the 38th Interscience Conference on Antimicrobial Agents and Chemotherapy (New York). San Diego, California: American Society for Microbiology. 1998.

20. Sutcliffe J, Tait-Kamradt A, Wondrack L. Streptococcus pneumoniae and Streptococcus pyogenes resistant to macrolides but sensitive to clindamycin: a common resistance pattern mediated by an efflux system. Antimicrob Agents Chemother 1996; 40: 1817-1824.

21. Nguyen L, Levy D, Ferroni A, Gehanno P, Berche P. Molecular epidemiology of Streptococcus pyogenes in an area where acute pharyngotonsillitis is endemic. J Clin Microbiol 1997; 35: $2111-2114$

22. Davies HD, McGeer A, Schwartz B et al. Invasive group A streptococcal infections in Ontario, Canada. $N$ Engl $\mathrm{J} \mathrm{Med}$ 1996; 335: 547-554.

23. Musser JM, Hauser AR, Kim MH, Schlievert PM, Nelson K, Selander RK. Streptococcus pyogenes causing toxic-shock-like syndrome and other invasive diseases: Clonal diversity and pyrogenic exotoxin expression. Proc Natl Acad Sci USA 1991; 88: $2668-2672$.

24. Stanley J, Linton D, Desai M, Efstratiou A, George R. Molecular subtyping of prevalent M serotypes of Streptococcus pyogenes causing invasive disease. $J$ Clin Microbiol 1995; 33 2850-2855

25. Muotiala A, Seppälä H, Huovinen P, Vuopio-Varkila J Molecular comparison of group A streptococci of T1M1 serotype from invasive and noninvasive infections in Finland. $J$ Infect Dis 1997; 175: 392-399.

26. Kiska DL, Thiede B, Caracciolo $\mathrm{J}$ et al. Invasive group A streptococcal infections in North Carolina: epidemiology, clinical features, and genetic and serotype analysis of causative organisms. J Infect Dis 1997; 176: 992-1000.

27. Nakashima K, Ichiyama S, Iinuma $\mathrm{Y}$ et al. A clinical and bacteriological investigation of invasive streptococcal infections in Japan on the basis of serotypes, toxin production, and genomic DNA fingerprints. Clin Infect Dis 1997; 25: 260-266.

28. Hauser AR, Stevens DL, Kaplan EL, Schlievert PM. Molecular analysis of pyrogenic exotoxins from Streptococcus pyogenes isolates associated with toxic shock-like syndrome. $J$ Clin Microbiol 1991; 29: 1562-1567.

29. Johnson DR, Stevens DL, Kaplan EL. Epidemiologic analysis of group A streptococcal serotypes associated with severe systemic infections, rheumatic fever, or uncomplicated pharyngitis. J Infect Dis 1992; 166: 374-384.

30. Reichardt W, Müller-Alouf H, Alouf JE, Köhler W. Erythrogenic toxins A, B and C: occurrence of the genes and exotoxin formation from clinical Streptococcus pyogenes strains associated with streptococcal toxic shock-like syndrome. FEMS Microbiol Lett 1992; 100: 313-322.

31. Black CM, Talkington DF, Messmer TO, Facklam RR, Hornes E, Olsvik Ø. Detection of streptococcal pyrogenic exotoxin genes by a nested polymerase chain reaction. Mol Cell Probes 1993; 7: 255-259.

32. Norrby-Teglund A, Newton D, Kotb M, Holm SE, Norgren M. Superantigenic properties of the group A streptococcal exotoxin speF (MF). Infect Immun 1994; 62: 5227-5233.

33. Tyler SD, Johnson WM, Huang JC et al. Streptococcal erythrogenic toxin genes: detection by polymerase chain reaction and association with disease in strains isolated in Canada from 1940 to 1991. J Clin Microbiol 1992; 30: 3127-3131.

34. Cleary PP, Kaplan EL, Handley JP et al. Clonal basis for resurgence of serious Streptococcus pyogenes disease in the 1980s. Lancet 1992; 339: 518-521.

35. Hsueh P-R, Wu J-J, Tsai P-J, Liu J-W, Chuang Y-C, Luh K-T. Invasive group A streptococcal disease in Taiwan is not associated with the presence of streptococcal pyrogenic exotoxin genes. Clin Infect Dis 1998; 26: 584-589.

36. Watanabe-Ohnishi R, Low DE, McGeer A et al. Selective depletion of $\mathrm{V} \beta$-bearing $\mathrm{T}$ cells in patients with severe invasive group A streptococcal infections and streptococcal toxic shock syndrome. J Infect Dis 1995; 171: 74-84.

37. Kotb M. Bacterial pyrogenic exotoxins as superantigens. Clin Microbiol Rev 1995; 8: 411-426.

38. Kamezawa Y, Nakahara T, Nakano S, Abe Y, Nozaki-Renard J, Isono T. Streptococcal mitogenic exotoxin $Z$, a novel acidic superantigenic toxin produced by a T1 strain of Streptococcus pyogenes. Infect Immun 1997; 65: 3828-3833.

39. Chaussee MS, Liu J, Stevens DL, Ferretti JJ. Genetic and phenotypic diversity among isolates of Streptococcus pyogenes from invasive infections. J Infect Dis 1996; 173: 901-908. 